\title{
Translocación Recíproca de los cromosomas 9 y 22 en los Genes BCR y ABL1: Leucemia Linfoide Aguda de Estirpe B Pediátrica
}

\section{Reciprocal Translocation of Chromosomes 9 and 22 of the BCR and ABL1 Genes: Paediatric B-Lineage Acute Lymphoblastic Leukaemia}

\begin{abstract}
Sayra Zooet Gonzalez-Mendez ${ }^{a}$, Daniel Medina-Bautista $^{b}$, Alma Barajas-Espinosa $^{c}$
Abstract:

Acute lymphoblastic leukaemia (ALL) is the most common malignancy neoplasm in children, it represents 70 to $80 \%$ of all leukaemias and develops most frequently between 2 and 5 years of life. ALL is characterized by uncontrolled proliferation and accumulation of immature white blood cells in the bone marrow. A subtype of ALL is the B lineage (ALL-B), characterized by the uncontrolled growth and accumulation of lymphoblasts as a result of genetic alterations of B cells. Among the associated genetic aberrations, we can find reciprocal translocation, although this is rare in ALL-B; specifically, the translocation $\mathrm{t}$ [9; 22] [q34; q11.2], that gives rise to the BCR-ABL1 oncoprotein which causes uncontrolled proliferation of leukemic cells. In conclusion, ALL is the most common paediatric neoplasm and its correlation with some genetic translocations, specifically the $\mathrm{t}[9 ; 22]$ [q34; $\mathrm{q} 11.2]$ translocation, may serve as a diagnostic tool or as a therapeutic target in the future.
\end{abstract}

Keywords:

Leukemia, Translocation, Oncoprotein, Philadelphia Chromosome, Lymphoblasts

\section{Resumen:}

La leucemia linfoblástica aguda (LLA) es la neoplasia maligna más común en los niños, está representa del 70 al $80 \%$ de todas las leucemias y se desarrolla con mayor frecuencia entre los 2 y 5 años de vida. LLA es caracterizada por una proliferación descontrolada y acumulación de glóbulos blancos inmaduros en la médula ósea. Un subtipo de LLA es la estirpe B (LLA-B), la cual se caracteriza por el crecimiento y acumulación descontrolada de linfoblastos como resultado a las alteraciones genéticas de células B. Entre las aberraciones genéticas asociadas, se encuentra la translocación recíproca, aunque esta es poco frecuente en la LLA-B, específicamente la translocación t[9;22] [q34;q11.2], que da como resultado la oncoproteína BCR-ABL1 la cual provoca una proliferación descontrolada de células leucémicas. En conclusión, la LLA es la neoplasia pediátrica más común y su correlación con algunas translocaciones genéticas, específicamente la translocación t[9;22] [q34;q11.2], puede fungir como herramienta diagnóstica o como blanco terapéutico en un futuro.

\section{Palabras Clave:}

Leucemia, Translocación, Oncoproteína, Cromosoma Filadelfia, Linfoblastos

\section{Introducción}

Cáncer es un término genérico para designar tal vez unas 200 distintas entidades. Desde el punto de vista biológico, es un trastorno esencialmente genético y caracterizado por un desequilibrio entre la proliferación y los mecanismos normales de muerte celular ${ }^{1}$. Si bien existen diversos tipos de cáncer, tales como próstata, pulmón, mama, garganta, etc. La leucemia es un tipo de cáncer que suele afectar a los glóbulos blancos, dicha neoplasia suele dividirse en dos tipos principales, aguda y crónica, los cuales a su vez se subdividen en linfoblástica y mieloide.

\footnotetext{
${ }^{a}$ Universidad Autónoma del Estado de Hidalgo, Email: go286332@uaeh.edu.mx

b Universidad Autónoma del Estado de Hidalgo, Email: me434191@uaeh.edu.mx

Autor de Correspondencia, Universidad Autónoma del Estado de Hidalgo, https://orcid.org/0000-0002-3961-3280, Email: alma_barajas@uaeh.edu.mx
} 
La leucemia linfoblástica aguda (LLA) es la neoplasia maligna más común en la población pediátrica, representando del 70 al $80 \%$ de todas las leucemias. Existe una incidencia mayor en varones, y típicamente se diagnostica entre los 2-5 años de vida ${ }^{2,3}$. En México se diagnostican cerca de 58 casos de LLA por millón de población infantil, colocando a dicha enfermedad como la segunda causa de muerte pediátrica en nuestro país ${ }^{4}$.

En esta revisión bibliográfica se analizarán las causas celulares y moleculares de la LLA, con énfasis sobre sus bases genéticas.

\section{Leucemia Linfoblástica Aguda (LLA): Etiología}

Como se mencionó anteriormente, la leucemia es el cáncer de los glóbulos blancos, en los cuales se han detectado mutaciones de células madre hematopoyéticas de la línea linfoide (Figura 1). Dicha enfermedad es linfoproliferativa sistémica y consiste de un grupo de entidades oncohematológicas de evolución rápida, clínica y biológicamente heterogéneas, caracterizadas por una proliferación descontrolada y acumulación de glóbulos blancos inmaduros en la médula ósea (MO), que pueden entrar a la circulación sanguínea periférica e infiltrarse a otros tejidos. La LLA es una neoplasia de los precursores linfoides, los linfoblastos; por lo tanto, se ven afectadas tanto las células B y las T.

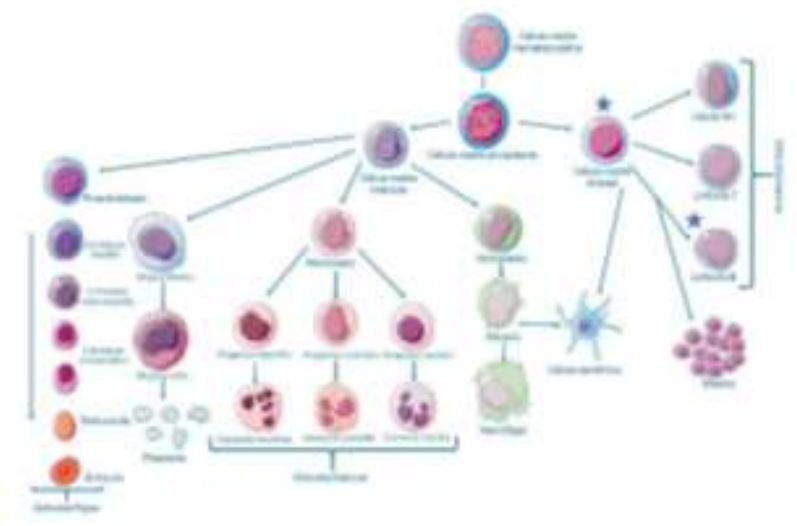

Figura 1. Linaje de células formadas por la hematopoyesis. En este diagrama se visualizan las diferentes células sanguíneas con un ancestro en común, la célula madre hematopoyética, que se diferencia a célula madre pluripotente y da lugar a la célula madre linfoide, de la cual se derivarán diversos glóbulos blancos, incluyendo el tipo B y T. Las células afectadas en la LLA-B son aquellas cuyos nombres están marcados con una estrella.

Debido a esto, desde el 2016 la OMS clasifica a las LLA en leucemia/linfoma linfoblástica B y T. El 80-85\% de todos los casos de LLA tienen su origen en los precursores de las células B, de acuerdo con las estirpes B o T cada uno de éstas tiene una amplia variedad de subtipos de LLA en esta ocasión nos enfocaremos en la leucemia linfoblástica aguda de estirpe B (LLA-B $)^{5-7}$. La LLA-B se caracteriza por el crecimiento y acumulación descontrolado de linfoblastos debido a las alteraciones genéticas de células $\mathrm{B}$ que bloquean la diferenciación celular. Entre dichas alteraciones genéticas de la LLA-B podemos encontrar la adquisición de una serie de aberraciones genéticas en las enzimas que altera la maduración normal de estas células debido a reordenamientos cromosómicos estructurales, como son las aneuploidías, rearreglos estructurales, translocaciones, inversiones, deleciones, monosomías y trisomías ${ }^{4,5}$. Las alteraciones más frecuentes en LLA-B son las translocaciones recíprocas, como $\mathrm{t}[12 ; 21]$ [p13;q22), $\mathrm{t}[1 ; 19]$ [q23;p13), y t[17;19] [q22;p13). En esta revisión nos enfocaremos en una translocación recíproca poco frecuente en la LLA-B, denominada como la translocación t[9;22] [q34;q11.2] (Figura 2), que da como resultado el cromosoma Filadelfia, el cual produce la oncoproteína BCR-ABL1, que presenta actividad tirosina quinasa relativamente alta y que ha sido asociada con provocar proliferación descontrolada de linfoblastos inmaduros de tipo B en la LLA-B pediátrica ${ }^{8}$.

\section{Cromosoma Filadelfia}

El cromosoma Filadelfia (Ph), generado por la translocación recíproca $\mathrm{t}[9 ; 22]$ [q34; q11] da como resultado la oncoproteína de fusión BCR-ABL1, detectable en el 2-5\% de los niños con LLA-B ${ }^{9}$. La BCR-ABL1 tiene actividad de tirosina quinasa, funciona de manera constitutiva, y modula vías de transducción de señales que conducen a la proliferación anormal de la médula ósea y la transformación maligna en la enfermedad ${ }^{10,11}$.

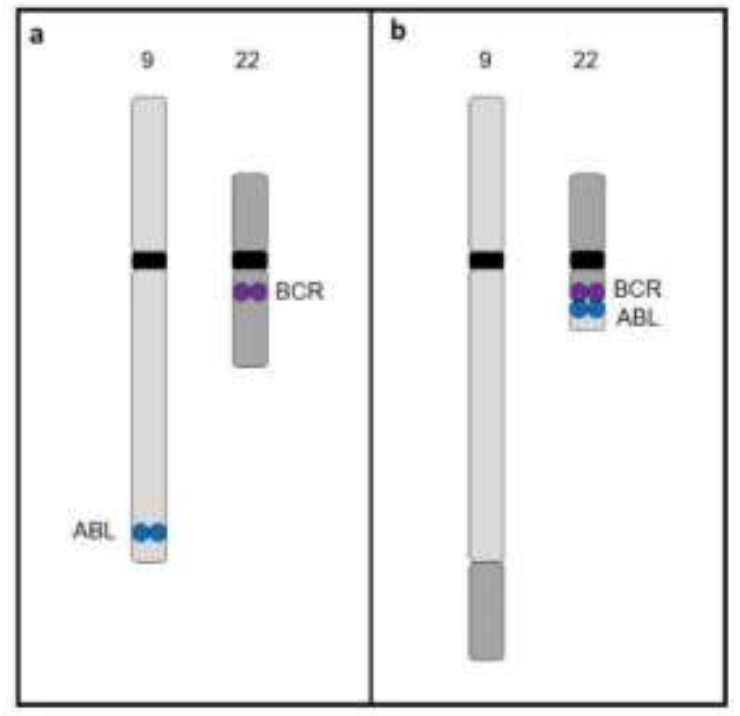

Figura 2. Diagrama representativo de la translocación recíproca t[9;22] [q34;q11.2], correspondiente a la LLA- 
B. En el panel (a) se representan los dos cromosomas ( 9 y 22) con sus centrómeros (banda negra) y los genes ABL y BCR en su posición fisiológicamente normal. A través de la translocación del segmento inferior (brazo q) de ambos cromosomas, el segmento del cromosoma número 9 con el gel ABL se yuxtaposiciona con el gen BCR correspondiente en el cromosoma número 22 (b). Debido a dicha translocación, la oncoproteína, BCR-ABL, se logra producir.

Debido a su correlación tan estrecha con LLA-B, la BCRABL1 se considera una oncoproteína, la cual es el resultado traduccional de la fusión del gen de la cinasa de Abelson (ABL, también conocido como ABL1) localizado en el cromosoma 9 con la región de clúster de punto de ruptura (BCR, del inglés: breakpoint cluster region) del cromosoma 22 (Figura 2). El punto de ruptura en el cromosoma 9 se localiza entre los exones a1, b1 y a2, mientras que los puntos de ruptura en el cromosoma 22 ocurren dentro del BCR, que consta de 23 exones dando como resultado varios genes de fusión distintos, usualmente los puntos de interrupción en BCR se localizan entre los exones b1-b5 en la llamada región de clúster de punto de ruptura principal (M-bcr). Más aún, se ha demostrado que existen otras dos regiones de clúster de ruptura menos frecuentes: menor (m-bcr), localizada entre los exones e1-e2; y micro ( $\mu$-bcr) localizada entre los exones e19-e $20^{9,10}$.

Por lo tanto, dependiendo del punto de ruptura de ABL1, la proteína de fusión tendrá distintos pesos moleculares: 190 KDa para m-bcr, 210 KDa para M-bcr y $230 \mathrm{KDa}$ para $\mu$-bcr. En la Leucemia Linfoblástica Aguda de estirpe B con cromosoma Filadelfia positivo (LLA-B $\mathrm{Ph}+$ ), la proteína BCR-ABL1 tiene un peso molecular de $190 \mathrm{KDa}$, resultado del reordenamiento del exón e1 del cromosoma 22 con el exón a2 del cromosoma 9 (e1a2 BCR-ABL) ${ }^{9,12}$.

\section{Efecto de BCR-ABL1}

Las proteínas tirosina-quinasas son conocidas por tener un papel fundamental en los procesos de transducción de señales de proliferación y diferenciación celular. Tales eventos se encuentran alterados en la LLA-B Ph+ debido a que la expresión aberrante de la oncoproteína BCR-ABL1 activa los mecanismos de proliferación de las células hematoprogenitoras, sobrepasando así mecanismos de regulación y provocando un ritmo de división descontrolado en las células oncogénicas.

La oncoproteína BCR-ABL1 otorga ventajas de supervivencia y proliferación a las células hematopoyéticas debido a la activación de varias vías de señalización ${ }^{13}$. Se ha demostrado que la actividad tirosina quinasa desregulada de BCR-ABL es necesaria y suficiente para mantener el fenotipo leucémico y, que la activación de la tirosina quinasa $\mathrm{ABL}$ es un evento primario en la génesis de la LLA-B ${ }^{14}$.
Se puede argumentar que la tirosina quinasa de fusión BCRABL1 causa inestabilidad genómica de manera directa, activa la vía de la proteína RAS, aumenta la actividad transcripcional a través del reclutamiento de STAT y disminución de la apoptosis mediante la activación de la ruta PI3K/AKT (Figura 3) ${ }^{15}$.

\section{Vías de señalización}

La proteína $\mathrm{ABL}$ se desplaza entre el núcleo y el citoplasma; sin embargo, cuando se fusiona con BCR, la oncoproteína pierde esta propiedad y se retiene principalmente en citoplasma, donde interactúa con la mayoría de las proteínas implicadas en la vía de señalización oncogénica. La actividad quinasa se activa constitutivamente por la yuxtaposición de BCR, favoreciendo así la dimerización o tetramerización y la subsiguiente autofosforilación. Esto aumenta el número de residuos de fosfotirosina en BCRABL y, como consecuencia, los sitios de unión para los dominios $\mathrm{SH} 2$ de otras proteínas ${ }^{14}$.

Las interacciones anormales entre la oncoproteína BCR-ABL y otras moléculas citoplasmáticas (Figura 3) conducen a la interrupción de procesos celulares clave, perturbación de la proteína quinasa activada por mitógeno Ras (MAPK) que conduce a una mayor proliferación, la vía de la quinasa Janus (JAK) activada por -STAT que lleva a una actividad transcripcional alterada y la vía de la fosfoinositol 3-quinasa (PI3K) / AKT resultando en una disminución de la apoptosis. Los efectos mitogénicos de BCR-ABL requieren la activación de: la vía PI3K /AKT, que promueve la supervivencia celular por fosforilación e inactivación de Bad, evitando así la apoptosis; la vía de la quinasa regulada por señales extracelulares Ras / Raf / MEK que participa en la regulación del ciclo celular; y la regulación positiva mediada por STAT5 ${ }^{14,16}$.

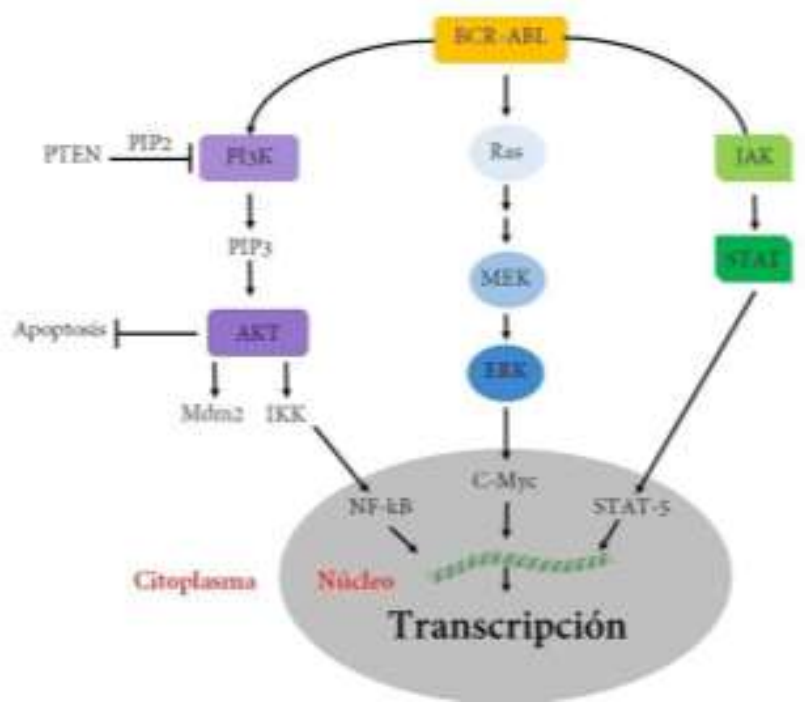


Figura 3. Vías de señalización celulares activadas por BCR-ABL. Entre las moléculas afectadas por la oncoproteína BCR-ABL, se encuentra la proteína quinasa activada por mitógeno Ras (MAPK) que conduce a una mayor proliferación, la vía de la quinasa Janus (JAK) activada por -STAT que lleva a una actividad transcripcional alterada y la vía de la fosfoinositol 3-quinasa (PI3K) / AKT que promueve la supervivencia celular vía inhibición de la apoptosis.

La activación de la vía PI3K/AKT por BCR-ABL1 promueve la proliferación celular (Figura 3) y tiene efectos anti apoptóticos al mediar la degradación de inhibidores del ciclo celular como p27kip $1^{16}$ además de promover la activación de factores de transcripción como NF-kB ${ }^{17}$. Por otro lado, la vía Ras/Raf/MEK/ERK da lugar a la activación de expresiones genéticas cuyos productos aumentan la proliferación celular e inhiben la apoptosis ${ }^{18}$. Más aún, la vía JAK/STAT activa la transcripción de genes anti apoptóticos como STAT5 ${ }^{19}$ que, a su vez, regula positivamente genes importantes para la supervivencia y proliferación de tumores hematopoyéticos cuando se activa de manera persistente en las células tumorales 20

\section{CONCLUSIÓN}

En conclusión, la leucemia linfoblástica aguda de estirpe B (LLA-B) pediátrica tiene una genética muy diversa, un ejemplo de esto son las translocaciones, que resultan en proteínas de fusión con funciones anormales y potentes propiedades oncogénicas. De acuerdo con lo investigado, la LLA-B $\mathrm{Ph}+$ es el resultado de una translocación cromosómica de los cromosomas 9 y 22 entre los genes BCR y ABL cuyo producto es una proteína tirosina-quinasa con actividad constitutiva. El gen de fusión BCR-ABL1 desencadena la expresión aberrante de las enzimas que regulan el proceso de señalización intracelular, y es gracias a la actividad tirosina quinasa persistente que BCR-ABL1 causa una inestabilidad genómica, siendo esta la responsable de alteraciones de las vías de señalización implicadas en la apoptosis, proliferación celular y regulación del ciclo celular, conllevando a la proliferación de células oncogénicas. La alta tasa de multiplicación celular y de reordenamientos genéticos son características que favorecen la aparición de mutaciones a nivel del ADN y promueven la transformación celular hacia un estado maligno.

\section{Agradecimientos}

Las figuras fueron adaptadas de Servier Medical Art, https://smart.servier.com/, y empleadas bajo Creative Commons Attribution 3.0.
1. Granados García M. Oncología y Cirujia. Bases y Principios. 1st ed. Manual Moderno; 2013.

2. Caniza MA, Jiménez de Samudio A, Samudio M. Factores de riesgo asociados a la sobrevida en niños y adolescenes con leucemia linfoblástica aguda. Pediatr (Asunción). 2016;43(1):18-26.

3. Masetti R. Avances en la terapia de la leucemia infantil. Ambiociencias. 2018:3021:5. doi:10.18002/ambioc.v0i0.5554

4. Zapata-Tarres M, Sánchez-Huerta J, Angeles-Floriano $\mathrm{T}$, et al. Identificación de alteraciones moleculares en pacientes pediátricos con diagnóstico de leucemia aguda. Rev Mex Hematol. 2017;18(2):47-57.

5. Fernández Moriano C. TISAGENLECLEUCEL ( $\nabla$ KYMRIAH®, NOVARTIS) EN LEUCEMIA LINFOBLÁSTICA AGUDA DE CÉLULAS B Y LINFOMA DIFUSO DE CÉLULAS GRANDES B. Med Clin (Barc). 2019;43(422):1-16. doi:10.1016/j.medcli.2020.02.011

6. Jiménez-Morales S, Hidalgo-Miranda A, Ramírez-Bello J. Leucemia linfoblástica aguda infantil: una aproximación genómica. Bol Med Hosp Infant Mex. 2017;74(1):13-26. doi:10.1016/j.bmhimx.2016.07.007

7. Ríos-Hernández KS, Rey-Vargas L, De los Reyes I, et al. Leucemia linfoide aguda B biclonal en un paciente pediátrico: reporte de caso en el servicio de Oncopediatría del Hospital Universitario San Ignacio. Univ Medica. 2020;61(1):84-91. doi:10.11144/javeriana.umed61-1.bicl

8. Verduzco Aguirre HC, Leonardo VR, López Ariza B. Leucemia linfoblástica aguda hiperdiploide en niños. Rev Hematoll. 2012;13(4):172-176

http://www.imbiomed.com.mx/1/1/articulos.php?method=showDetail \&id_articulo=94911\&id_seccion=3175\&id_ejemplar=9278\&id_revist $\mathrm{a}=191$

9. Piedimonte M, Ottone T, Alfonso V, et al. A rare BCR-ABL1 transcript in Philadelphia-positive acute myeloid leukemia: Case report and literature review. BMC Cancer. 2019;19(1):1-6. doi:10.1186/s12885019-5265-5

10. Savona M, Talpaz M. Getting to the stem of chronic myeloid leukaemia. Nat Rev Cancer. 2008;8(5):341-350. doi:10.1038/nrc2368

11. Vardiman JW. Chronic myelogenous leukemia, BCR-ABL1+. Am J Clin Pathol. 2009;132(2):250-260. doi:10.1309/AJCPUN89CXERVOVH

12. Weisberg E, Manley PW, Cowan-Jacob SW, Hochhaus A, Griffin JD. Second generation inhibitors of BCR-ABL for the treatment of imatinibresistant chronic myeloid leukaemia. Nat Rev Cancer. 2007;7(5):345356. doi:10.1038/nrc2126

13. Kurosu T, Nagao T, Wu N, Oshikawa G, Miura O. Inhibition of the PI3K/Akt/GSK3 pathway downstream of BCR/ABL, Jak2-V617F, or FLT3-ITD downregulates DNA damage-induced Chk1 activation as well as $\mathrm{G} 2 / \mathrm{M}$ arrest and prominently enhances induction of apoptosis. PLoS One. 2013;8(11). doi:10.1371/journal.pone.0079478

14. Cilloni D, Saglio G. Molecular pathways: BCR-ABL. Clin Cancer Res. 2012;18(4):930-937. doi:10.1158/1078-0432.CCR-10-1613

15. Radich JP. The Biology of CML blast crisis. Hematology Am Soc Hematol Educ Program. 2007;(Cml):384-391. doi:10.1182/asheducation-2007.1.384

16. Andreu EJ, Lledó E, Poch E, et al. BCR-ABL induces the expression of Skp2 through the PI3K pathway to promote p27Kip1 degradation and proliferation of chronic myelogenous leukemia cells. Cancer Res. 2005;65(8):3264-3272. doi:10.1158/0008-5472.CAN-04-1357

\section{Referencias}


17. Gorre ME, Mohammed M, Ellwood K, et al. Gore Imatinib clinical resistance Science 2001. Science (80- ). 2001;293(5531):876-880. papers://17e5e17d-39c3-4c1f-97e1-f35fdc09783f/Paper/p1934

18. Chang F, Steelman LS, Lee JT, et al. Signal transduction mediated by the Ras/Raf/MEK/ERK pathway from cytokine receptors to transcription factors: Potential targeting for therapeutic intervention. Leukemia. 2003;17(7):1263-1293. doi:10.1038/sj.leu.2402945

19. Gallipoli P, Cook A, Rhodes S, et al. JAK2/STAT5 inhibition by nilotinib with ruxolitinib contributes to the elimination of CML CD34+ cells in vitro and in vivo. Blood. 2014;124(9):1492-1501. doi:10.1182/blood-2013-12-545640

20. Yu H, Pardoll D, Jove R. STATs in cancer inflammation and immunity: A leading role for STAT3. Nat Rev Cancer. 2009;9(11):798-809. doi: $10.1038 /$ nrc2734 